BARBOSA, I.L.A. et al. Ganho de peso em bovinos em confinamento utilizando enrofloxacino e polivacinas. PUBVET, Londrina, V. 5, N. 8, Ed. 155, Art. 1049, 2011.

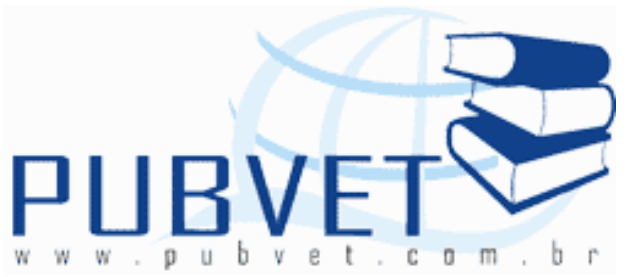

PUBVET, Publicações em Medicina Veterinária e Zootecnia.

\title{
Ganho de peso em bovinos em confinamento utilizando enrofloxacino e polivacinas
}

\section{Isadora Langoni Amorim Barbosa ${ }^{1 *}$, Mariela Silva Moura ${ }^{1}$, João Paulo Rodrigues Bueno $^{1,}$ Francisco Sales de Resende Carvalho ${ }^{2}$, Carlos Roberto Silva ${ }^{3}$}

${ }^{1}$ Graduando em Medicina Veterinária, Universidade Federal de Uberlândia. Uberlândia, MG.

${ }^{2}$ Docente da Faculdade de Medicina Veterinária, Universidade Federal de Uberlândia. Uberlândia, MG.

${ }^{3}$ Médico Veterinário

*Autor para correspondência: Rua Ceará, s/n, Bloco 2D, CEP 38400-902, Uberlândia, MG, Brasil. E-mail: isalangoni@hotmail.com

\section{Resumo}

Para um bom desenvolvimento de um rebanho, é importante o uso de vacinas e medicamentos como forma de minimizar algumas infecções mais freqüentes em confinamento, como problemas respiratórios, além de envolver a seleção de animais, alimentação de qualidade e infra-estrutura adequada na propriedade. Objetivou-se com este trabalho avaliar o ganho de peso nos animais confinados, que foram submetidos ao uso do medicamento metafilático Enrofloxacino e polivacinas. Foram selecionados 420 bovinos, machos, não castrados, da raça Nelore entre 24 e 30 meses de idade, alocados em dois currais da Fazenda Nova, Uberlândia, Minas Gerais. Foram pré- 
BARBOSA, I.L.A. et al. Ganho de peso em bovinos em confinamento utilizando enrofloxacino e polivacinas. PUBVET, Londrina, V. 5, N. 8, Ed. 155, Art. 1049, 2011.

selecionados 500 animais com pesos entre 380 e $550 \mathrm{~kg}$, e destes foram retirados 420 animais, que foram marcados como lote 34 , alocados no curral 1 e lote 38 alocados no curral 2. Após a formação do lote 34 (tratamento metafilático com Enrofloxacino) e lote 38 (controle), novamente efetuou-se nova divisão dentro de cada lote, no qual foram formados três grupos com 70 bovinos dentro de cada lote, também levando em consideração o peso dos animais. Os bovinos foram submetidos à pesagem no dia 0 , e posteriormente foram pesados nos dias 30, 60, sempre levando em consideração o regime hídrico e alimentar. Após o abate $100 \%$ dos pulmões dos animais do grupo tratado e do grupo controle foram avaliados para visualização de alterações patológicas visíveis, que pudessem ser decorrentes de infecções pulmonares. Também foi efetuada a análise econômica dos ganhos obtidos, em comparação do lote/grupo, durante o período experimental. Com esse trabalho podemos concluir que o Enrofloxacino pode ser usado com sucesso como metafilático em animais confinados, proporcionando a redução de doenças respiratórias e aumentando o ganho de peso dos animais.

Palavras-chave: Bovinos, custos, ganho de peso, infecções pulmonares, tratamento metaprofilático.

\section{Weight gain in feedlot cattle using enrofloxacin and several vaccines.}

\section{Abstract}

For a good herd development, it is important to use vaccines and medicines to minimize some infections more frequent in confinement, such as respiratory problems. It involves animals selection, feed quality and adequate property infrastructure as well. This study aimed to evaluate weight gain in contained animals, that were submitted to the medication and metaprofilático Enrofloxacino e polivacinas. We selected 420 cattle, bulls, Nellore between 24 and 30 months of age, divided into two corrals at "Fazenda Nova", Uberlandia, Minas Gerais. Were pre-selected 500 animals weighing between 380 and 550 $\mathrm{kg}$, and selected 420 animals from the previous group, which were marked as 
BARBOSA, I.L.A. et al. Ganho de peso em bovinos em confinamento utilizando enrofloxacino e polivacinas. PUBVET, Londrina, V. 5, N. 8, Ed. 155, Art. 1049, 2011.

Lot 34, placed in a corral 1 and lot 38 allocated in the corral 2. After setting batch 34 (with metafilático Enrofloxacino treatment) and batch 38 (control), again a new division for done within each group in which three groups were formed with 70 animals in each batch, also taking into account the weight animals. The cattle were weighed on day 0 , and then were weighed on days 30,60 , always taking into consideration the food and water regime. After slaughter $100 \%$ of animals' lungs of the treated group and control group were evaluated for visualization of pathological changes visible, which could be due to lung infections. It was also performed the analysis of economic gains, compared batch / group during the experimental period. With this work we can conclude that Enrofloxacino can be used successfully as metaprofilático in confined animals, providing a reduction of respiratory diseases and increasing the weight gain of animals.

Keywords: Bovines, costs, lung infections, metaprofilático treatment, weight gain.

\section{Introdução}

"É chamado de "confinamento" o sistema de criação de bovinos em que lotes de animais são encerrados em piquetes ou currais com área restrita, e onde os alimentos e água necessários são fornecidos em cochos" (CARDOSO, 2000).

O Brasil atualmente possui o maior rebanho bovino comercial do mundo, com cerca de 160 milhões de cabeças e é o segundo maior produtor mundial de carcaças bovinas com um total de 7321 toneladas atuais. A pecuária de corte ocupa lugar de destaque na economia do país com um faturamento anual em torno de 14 bilhões de dólares e ocupação de 7 milhões de pessoas. Esse crescimento está ocorrendo, pelo fato que o produtor procura uma maneira de aumentar seu capital de giro e diminuir o preço da arroba produzida, em conseqüência encurta o processo de abate, e assim obtêm um melhor retorno do capital investido. "Muitos confinamentos no Brasil estão 
BARBOSA, I.L.A. et al. Ganho de peso em bovinos em confinamento utilizando enrofloxacino e polivacinas. PUBVET, Londrina, V. 5, N. 8, Ed. 155, Art. 1049, 2011.

localizados nas regiões do centro-oeste (MS, MT e GO) do país e oeste Paulistano" (FARIA, 2007).

É importante ressaltar que o processo de confinamento geralmente é realizado em épocas de seca, pelo motivo que este período apresenta uma escassez de forrageiras, podendo levar os animais à perda de peso. Estes bovinos são comercializados no pico da entressafra quando então tendem a alcançar melhores preços.

De acordo com Cardoso, et al (1996) o confinamento de bovinos por proprietários de rebanhos ou fazendeiros traz consigo as seguintes vantagens:

- Aumento da eficiência produtiva do rebanho, por meio da redução na idade de abate e melhor aproveitamento do animal produzido e capital investido nas fases anteriores

- Uso mais eficiente de mão-de-obra, maquinários e insumos; e flexibilidade de produção.

Para o manejo da alimentação dos bovinos confinados é necessária uma estrutura de logística, referente à compra, venda e armazenamento dos alimentos, pois esses são utilizados em grande escala, sendo os responsáveis em proporcionar um bom desempenho produtivo dos animais.

Atualmente nos grandes confinamentos, é inviável produzir volumosos em alta escala (há uma necessidade de grande área). Uma das saídas é utilizar as dietas de "alto grão" (10\% de volumosos/90\% de concentrados), adquirindo os concentrados no mercado (DUARTE, 2008).

Podemos ressaltar alguns fatores que comprometem o desempenho animal em conjunto, a produtividade ou lucratividade do sistema como: presença de lama nos currais, comprimento de cocho insuficiente, uso de alimentos de baixa palatabilidade, como a farinha de carne em proporção relativamente alta, picagem do capim verde a ser fornecido com muita antecedência à hora da refeição (esquenta e fermenta, perdendo paladar), animal sem boa conformação óssea e muscular, lotes com animais de porte, condição ou idade diferentes, excessiva movimentação dos animais, constante 
BARBOSA, I.L.A. et al. Ganho de peso em bovinos em confinamento utilizando enrofloxacino e polivacinas. PUBVET, Londrina, V. 5, N. 8, Ed. 155, Art. 1049, 2011.

presença de pessoas estranhas, alteração dos horários e forma de fornecimento de alimentos. (CARDOSO, 2000)

Segundo Brandini et al (1996), as principais doenças que acometem com mais freqüência os bovinos confinados são: Botulismo, Enterotoxemia, Dermatofilose, Timpanismo, Acidose Lática, Laminite, intoxicação por uréia, dermatomicose e problemas respiratórios.

É importante ressaltar o uso das vacinas e remédios para minimizar algumas infecções mais freqüentes em confinamento. Esse também é um processo que requer tecnologia avançada e adequada, que envolve a seleção de animais, alimentação de baixo custo e de boa qualidade, infra-estrutura adequada na propriedade (MELLO, 1996). Associado a esses fatores citados é importante um manejo calmo e tranqüilo nos animais que irão entrar em confinamento, ou mesmo que sejam manejados de rotina, já que as bactérias e vírus estão à espera de um momento de um simples stress, sejam estes ocasionados por gritos ou correrias durante a condução dos bovinos, o acompanhamento do ganho de peso diário dos animais, pois ele reflete se o manejo, como alimentação, água, sanidade do rebanho estão eficientes. Bons índices de ganho de peso sugerem menor tempo para o abate dos animais, e melhor retorno do capital investido.

Levando em consideração os problemas respiratórios e outros problemas que podem acometer os bovinos confinados, devido as mudança de manejo, este trabalho se propôs avaliar o uso da metafilaxia com Enrofloxacino, Bayer S.A, juntamente com um pacote de produtos Bayer (Baymec Prolong, Bayverm, Catosal) comparados com o segundo lote de animais que não receberam o tratamento metafilático, apenas o tratamento de rotina de confinamento.

\section{Material e métodos}

Foram selecionados 420 bovinos, machos, não castrados, da raça Nelore entre 24 e 30 meses de idade, alocados em dois currais da Fazenda Nova, Uberlândia, Minas Gerais. Os dados obtidos foram coletados na mesma 
BARBOSA, I.L.A. et al. Ganho de peso em bovinos em confinamento utilizando enrofloxacino e polivacinas. PUBVET, Londrina, V. 5, N. 8, Ed. 155, Art. 1049, 2011.

fazenda. Os animais foram selecionados de acordo com o peso e identificados de acordo com os padrões de Boas Práticas Clínicas.

Foram pesador em balança eletrônica e pré-selecionados 500 animais com pesos entre 380 e $550 \mathrm{~kg}$, e destes foram retirados 420 animais, que foram marcados como lote 34 , alocados no curral 1 e lote 38 alocados no curral 2, com fornecimento de água e alimentação à vontade para ambos.

Selecionados 420 bovinos, os mesmos foram distribuídos em dois lotes de 210 animais cada, obedecendo ao seguinte critério: os dois bovinos identificados, que apresentavam maior peso foram sorteados, um para cada lote. Este procedimento casualístico foi conduzido da mesma forma e sucessivamente, com dois bois de peso imediatamente abaixo, até identificação e sorteio dos dois últimos animais. Compostos os grupos, efetuose um sorteio para estabelecer-se um lote controle e um lote tratado.

Após a formação do lote 34 (tratado) e lote 38 (controle), novamente efetuou-se nova divisão dentro de cada lote, obedecendo ao mesmo critério supracitado, no qual foram formados três grupos com 70 bovinos dentro de cada lote, também levando em consideração o peso dos animais.

Formados os grupos, os animais do lote tratado (tratamento metafilático) foram divididos em três grupos de 70 animas que receberam os seguintes produtos de acordo com a recomendação do fabricante:

a) Grupo 1 - Lote 34 - Enrofloxacino ${ }^{1}$, pela via subcutânea, 30 ml/animal; Bayverm², pela via subcutânea, $10 \mathrm{ml} /$ animal; Baymec Prolong ${ }^{3}$, pela via subcutânea, $8 \mathrm{ml} /$ animal; Catosal ${ }^{4}$, pela via subcutânea, $10 \mathrm{ml}$ no D0 e D30; Vacina Biopoligen ${ }^{5}$, pela via subcutânea e Vacina Poli- $R^{6}$ também pela via subcutânea.

\footnotetext{
${ }^{1}$ Bayer- antibiótico de rápida recuperação.

${ }^{2}$ Bayer - tratamento e controle de helmintíases gastrintestinais e pulmonares.

${ }^{3}$ Bayer-parasiticida de amplo espectro.

${ }^{4}$ Bayer- atividade anabólica.

${ }^{5}$ Biogénesis Bagó- Vacina combinada viral e bacteriana, para a prevenção de quadros respiratórios, reprodutivos, nervosos entéricos.

${ }^{6}$ Vallée- vacina que protege contra as clostridioses
} 
BARBOSA, I.L.A. et al. Ganho de peso em bovinos em confinamento utilizando enrofloxacino e polivacinas. PUBVET, Londrina, V. 5, N. 8, Ed. 155, Art. 1049, 2011.

b) Grupo 2 - lote 34 - Enrofloxacino, pela via subcutânea. 30 ml/animal, Bayverm, pela via subcutânea, $10 \mathrm{ml} /$ animal; Baymec Prolong pela via subcutânea, $8 \mathrm{ml} /$ animal; Vacina Biopoligen, pela via subcutânea e Vacina Poli$\mathrm{R}$ também pela via subcutânea.

c) Grupo 3 - lote 34 - Enrofloxacino, pela via subcutânea, 30ml/animal; Bayverm, pela via subcutânea, $10 \mathrm{ml} /$ animal; Baymec Prolong, pela via subcutânea, $8 \mathrm{ml} /$ animal; Catosal, pela via subcutânea, $10 \mathrm{ml}$ no DO; Vacina Biopoligen, pela via subcutânea e Vacina Poli-R também pela via subcutânea.

Os animais do grupo controle curral 2-lote 38, receberam:

d) Grupo 1 - lote 38 - Lancer (abamectina), pela via subcutânea, $8 \mathrm{ml} /$ animal, Ricobendazole, pela via subcutânea, $10 \mathrm{ml} /$ animal; Vacina Biopoligen, pela via subcutânea e Vacina Poli-R também pela via subcutânea.

e) Grupo 2 - lote38 - Lancer (abamectina), pela via subcutânea, $8 \mathrm{ml} /$ animal; Ricobendazole, pela via subcutânea, $10 \mathrm{ml} /$ animal; Catosal, pela via subcutânea, $10 \mathrm{ml} /$ animal no D0, Vacina Biopoligen, pela via subcutânea e Vacina Poli-R também pela via subcutânea;

f) Grupo 3 - lote 38 - Lancer (abamectina), pela via subcutânea, $8 \mathrm{ml} /$ animal; Ricobendazole, pela via subcutânea, $10 \mathrm{ml} /$ animal; Catosal, pela via subcutânea, $10 \mathrm{ml} /$ animal no D0 e D30; Vacina Biopoligen, pela via subcutânea e Vacina Poli-R também pela via subcutânea.

Após a aplicação da medicação, os animais foram observados para verificação de reações adversas. Qualquer alteração clínica, reação adversa sistêmica ou local, morte dos animais, ou problemas clínicos que ocorressem após a aplicação do medicamento ou durante o experimento foram relatados nos diários do campo anexados ao relatório final do experimento.

Os bovinos foram submetidos à pesagem no dia 0 , e posteriormente foram pesados nos dias 30,60, sempre levando em consideração o regime hídrico e alimentar. Após o abate $100 \%$ dos pulmões dos animais do grupo tratado e do grupo controle foram avaliados para visualização de alterações patológicas visíveis, que pudessem ser decorrentes de infecções pulmonares adquiridas durante o período experimental. 
BARBOSA, I.L.A. et al. Ganho de peso em bovinos em confinamento utilizando enrofloxacino e polivacinas. PUBVET, Londrina, V. 5, N. 8, Ed. 155, Art. 1049, 2011.

Ao final do período foi calculado o ganho de peso individual de cada grupo/lote e estes dados foram submetidos ao programa INSTAT 1998, para análise estatística.

Também foi efetuada a análise econômica dos ganhos obtidos, em comparação do lote/grupo, durante o período experimental. Na tabela 1, são apresentados os preços e doses dos produtos utilizados para medicação e que compuseram o custo do tratamento para cada lote/grupo experimental.

Tabela 1. Custos dos produtos utilizados no experimento e que compuseram o custo dos tratamentos utilizados nos Lotes/grupos experimentais, Uberlândia, 2008.

\begin{tabular}{lccc}
\hline \multicolumn{1}{c}{ Produtos } & Apresentação & Custo (R\$) & $\begin{array}{c}\text { Preço da dose } \\
\text { (R\$) }\end{array}$ \\
\hline Catosal & $100 \mathrm{ml}$ & 55,00 & $5,5(10 \mathrm{ml})$ \\
Baymec & $1000 \mathrm{ml}$ & 245,00 & $1,96(8 \mathrm{ml})$ \\
Bayverm & $250 \mathrm{ml}$ & 24,00 & $0,96(10 \mathrm{ml})$ \\
\hline Enrofloxacino & $50 \mathrm{ml}$ & 30,00 & $18,00(30 \mathrm{ml})$ \\
Ricobendazole & $250 \mathrm{ml}$ & 24,00 & $0,96(10 \mathrm{ml})$ \\
\hline Lancer & $500 \mathrm{ml}$ & 22,00 & $0,32(8 \mathrm{ml})$ \\
Biopoligen & $50 \mathrm{ml}$ & 93,75 & 3,75 \\
Poli-R & $100 \mathrm{ml}$ & 16,83 & 0,51 \\
\hline
\end{tabular}

\section{Resultados e Discussão}

Os dados de peso do lote 34 (grupos 1,2 e 3) e lote 38 (grupos 1,2 e 3) são apresentados nas tabelas abaixo. Nas tabelas 2, 3 são apresentados os pesos médios no D0, D30 e D60, a análise estatística dos grupos dentro de cada lote e os respectivos custos de cada tratamento recebido pelos grupos experimentais dentro de cada lote experimental. 
BARBOSA, I.L.A. et al. Ganho de peso em bovinos em confinamento utilizando enrofloxacino e polivacinas. PUBVET, Londrina, V. 5, N. 8, Ed. 155, Art. 1049, 2011.

Tabela 2- Pesos dos animais experimentais Lote 34, ganho de peso no período, ganho de peso diário e custos por tratamento, Uberlândia, 2008.

\begin{tabular}{|c|c|c|c|c|c|c|}
\hline Grupos & $\begin{array}{l}\text { DO } \\
(\mathrm{kg})\end{array}$ & $\begin{array}{l}\text { D30 } \\
\text { (kg) }\end{array}$ & $\begin{array}{l}\text { D60 } \\
(\mathrm{Kg})\end{array}$ & $\begin{array}{l}\text { GP } \\
(\mathbf{K g})\end{array}$ & $\begin{array}{l}\text { GD } \\
(\mathbf{K g})\end{array}$ & $\begin{array}{l}\text { Custo } \\
\text { (R\$) }\end{array}$ \\
\hline 1 & 421,44 & 516,22 & 550.88 & $129,44 \pm 25,17^{a}$ & 2,157 & 36,18 \\
\hline 2 & 422,20 & 505,21 & 541,40 & $119,20 \pm 25,94 b c$ & 1,986 & 25,18 \\
\hline 3 & 423,81 & 514,62 & 548,41 & $124,60 \pm 20,27 a c$ & 2,076 & 30,68 \\
\hline
\end{tabular}

Letras iguais na mesma coluna não diferem entre si pelo teste de Tukey $(p>0,05)$ GP- Ganho de peso; GD- Ganho de peso diário; Custo- Custo dos medicamentos aplicados

A tabela 2 apresenta o ganho de peso médio do lote 34 , e os grupos 1 , 2 e 3 deste lote. Pode-se verificar que o grupo 1 teve uma ganho médio de $129,44 \mathrm{Kg}$, seguido pelo grupo 3 com 124,60, e o grupo 2 com 119,20 Kg durante todo o período experimental, sendo que o grupo 2 difere estatisticamente dos grupos 1 e 3, e ambos não diferem entre si.

Ao analisarmos a tabela 3 abaixo, verificamos os pesos do lote 38 e de seus grupos também discriminados 1,2 e 3 . Aqui podemos constatar que não houve diferença estatística entre os pesos dos três grupos. Nas tabelas 2 e 3 também são apresentados os custos individuais por tratamento para cada grupo dentro dos seus respectivos lotes. O grupo 1-L34 (lote 34), teve um custo de $R \$ 36,18$, seguido do grupo 2-L34 com $R \$ 25,18$ e $R \$ 30,68$ para o grupo 3-L34. Estes custos se devem principalmente ao uso do Enrofloxacino que contribui em $\mathrm{R} \$ 18,00$ para cada animal, outro fator que pesou no custo também foi a aplicação de uma ou duas doses de Catosal ( $R \$ 5,5$ por dose de $10 \mathrm{ml}$ ). É importante ressaltar que no grupo 1-L34 que recebeu o tratamento completo, ou seja, com o custo mais alto, o ganho de peso foi superior aos dos demais grupos deste lote. 
BARBOSA, I.L.A. et al. Ganho de peso em bovinos em confinamento utilizando enrofloxacino e polivacinas. PUBVET, Londrina, V. 5, N. 8, Ed. 155, Art. 1049, 2011.

Tabela 3 - Peso dos animais experimentais lote 38, ganho de peso no período, ganho de peso diário e custos por tratamento, Uberlândia, 2008.

\begin{tabular}{|c|c|c|c|c|c|c|}
\hline Grupos & $\begin{array}{l}\text { DO } \\
(\mathbf{K g})\end{array}$ & $\begin{array}{l}\text { D30 } \\
\text { (Kg) }\end{array}$ & $\begin{array}{l}\text { D60 } \\
(\mathrm{Kg})\end{array}$ & $\begin{array}{l}\text { GP } \\
(\mathbf{K g})\end{array}$ & $\begin{array}{l}\text { GD } \\
(\mathbf{K g})\end{array}$ & $\begin{array}{l}\text { Custo } \\
\text { (R\$) }\end{array}$ \\
\hline 1 & 425,27 & 495,42 & 540,11 & $114,44 \pm 18,25^{a}$ & 1,901 & 5,54 \\
\hline 2 & 427,03 & 494,92 & 542,44 & $115,41 \pm 16,93 a b$ & 1,923 & 12,34 \\
\hline 3 & 427,95 & 496,85 & 547,33 & $119,05 \pm 21,04 a b c$ & 1,984 & 19,14 \\
\hline
\end{tabular}

Letras iguais na mesma coluna não diferem entre si pelo teste de Tukey $(p>0,05)$ GP- Ganho de peso; GD- Ganho de peso diário; Custo- Custo dos medicamentos aplicados

Dando continuidade à análise, na tabela 4, logo abaixo, temos a comparação entre os lotes 34 e 38, levando em consideração o uso ou não de Enrofloxacino e o uso do Catosal uma vez ou duas vezes.

Tabela 4 - Comparação entre os Lote 34 e os grupos que receberam pacote Bayer com variações e Lote 38 (Pacote confinamento + grupos com Catosal e sem Catosal), Uberlândia, 2008.

\begin{tabular}{llllllllll}
\hline Grupos & $\mathbf{D O}$ & $\mathbf{D 3 0}$ & $\mathbf{D 6 0}$ & $\mathbf{G P}$ & $\mathbf{C u s t o}$ & $\mathbf{G D}$ & $\mathbf{G L}$ & $\mathbf{G L D}$ & $\mathbf{G R}$ \\
& $\mathbf{( K g )}$ & $\mathbf{( K g )}$ & $\mathbf{( K g )}$ & $\mathbf{( K g )}$ & $\mathbf{( R \$ )}$ & $\mathbf{( K g )}$ & $\mathbf{( K g )}$ & $\mathbf{( R \$ )}$ & $\mathbf{( R \$ )}$ \\
\hline $\mathbf{1 - L 3 4}$ & 421,44 & 516,22 & 550,88 & $129,44 \pm 25,17 \mathrm{a}$ & 36,18 & 10,39 & 5,506 & 33,00 & 13,36 \\
$\mathbf{3 - L 3 8}$ & 427,95 & 496,85 & 547,33 & $119,05 \pm 21,04 \mathrm{~b}$ & 16,54 & & & & \\
$\mathbf{3 - L 3 4}$ & 423,81 & 514,62 & 548,41 & $124,60 \pm 20,27 \mathrm{a}$ & 30,68 & 9,19 & 4,870 & 29,22 & 9,52 \\
$\mathbf{2 - L 3 8}$ & 427,03 & 494,92 & 542,44 & $115,41 \pm 16,93 \mathrm{~b}$ & 11,04 & & & & \\
2-L34 & 422,20 & 505,21 & 541,40 & $119,20 \pm 25,94 \mathrm{a}$ & 25,18 & 4,76 & 2,591 & 15,55 & $-4,09$ \\
1-L38 & 425,27 & 495,42 & 540,11 & $114,44 \pm 18,25 \mathrm{ab}$ & 5,54 & & & & \\
\hline
\end{tabular}

Letras iguais na mesma coluna não diferem entre si pelo teste de Tukey $(p>0,05)$

GP- Ganho de peso; GD- Ganho de peso diário

Custo- Custo dos medicamentos aplicados

GL- Ganho Líquido com $53 \%$ de rendimento de carcaça

GLD- Ganho Líquido em dinheiro (Custo de R $\$ 90,00$ por arroba)

GR- Ganho Real após a comparação entre os tratamentos (GLD- Diferença entre os custos) 
BARBOSA, I.L.A. et al. Ganho de peso em bovinos em confinamento utilizando enrofloxacino e polivacinas. PUBVET, Londrina, V. 5, N. 8, Ed. 155, Art. 1049, 2011.

Nesta tabela podemos verificar que ao compararmos o grupo 1-L34 (Enrofloxacino, Catosal 2x, Baymec, Bayverm) com o lote 3-L38 (Lancer, Ricobendazole, Catosal $2 x$ ), houve diferença estatística entre os tratamentos $(p<0,05)$, o ganho diferencial GD foi de $10,39 \mathrm{Kg}$, seguido do ganho líquido $(5,506 \mathrm{Kg})$ o que proporcionou um ganho de $\mathrm{R} \$ 33,00$ com este peso a mais por animal. Ao efetuarmos a diferença de custos entre os lotes e descontarmos o quanto o lote 1-L34 rendeu a mais, temos um ganho final de $R \$ 13,36$ por animal, e um total de $\mathrm{R} \$ 935,20$ (70 animais do grupo 1-L34) a mais para o grupo que recebeu o Pacote Bayer completo em relação ao tratamento convencional do confinamento com aplicação de duas doses de $10 \mathrm{ml}$ de Catosal. Ainda na mesma tabela temos a comparação entre os grupos 3-L34 e 2-L38 e também podemos verificar que o uso do Enrofloxacino, Baymec, Bayverm e Catosal 1x, proporcionou um ganho médio $(124,60 \mathrm{Kg})$ superior ao do grupo 2-L38 $(115,41 \mathrm{Kg})$ pacote confinamento + Catosal $1 \mathrm{x}$, sendo estatisticamente diferente $(p<0,05)$. Já ao efetuarmos a análise do custo verificamos que mesmo havendo um ganho de peso superior, o tratamento do grupo 3-L34 levou um ganho positivo de $R \$ 9,2$ por animal, e um total de $R \$$ 666,40 em relação ao grupo 2-L38 (70 animais).

Ao compararmos os grupo 2-L34 e 1-L38, verificamos que não há diferença estatística entre os lotes, mas ao compararmos os custos em relação ao ganho líquido de $2,59 \mathrm{Kg}$ apresentado pelo grupo 2-L34 verificamos que o valor negativo por animal foi de - $R \$ 4,09$, dando um total - $R \$ 286,30$ (70 animais) para o grupo em relação ao grupo $1 \mathrm{~L} 38$.

A tabela 5 a seguir, faz a comparação geral entre os lotes 34 e 38, não levando em consideração os grupos individuais de cada lote. Nesta tabela podemos observar que os animais tratados com pacote Bayer apresentaram um ganho médio de $124,41 \mathrm{Kg}$ contra $116,54 \mathrm{Kg}$ do lote 38 , sendo $(p<0,05)$. Mas ao efetuarmos à análise econômica entre os lotes verificamos que os animais que receberam o pacote Bayer apresentaram um ganho líquido negativo de - $R \$ 5,44$ por animal, dando um total de $-R \$ 1142,40$ para o lote de 210 animai em relação ao custo do tratamento de rotina colocado no 
BARBOSA, I.L.A. et al. Ganho de peso em bovinos em confinamento utilizando enrofloxacino e polivacinas. PUBVET, Londrina, V. 5, N. 8, Ed. 155, Art. 1049, 2011.

confinamento, já levando em consideração que no lote 38 houve aplicação de Catosal nos animais dos grupos 2 e 3 do lote 38 .

Através desta observação podemos inferir que o Enrofloxacino cumpriu seu papel como metafilático, uma vez que os animais do lote 34 não apresentaram na avaliação dos pulmões nenhuma lesão que caracterizasse quadro pneumônico. Importante ressaltar também que os animais que apresentaram menor ganho de peso do lote 38, foram os animais que apresentaram presença de lesões pneumônicas.

O lote que recebeu Enrofloxacino, mesmo havendo variações dentro dos grupos formados neste lote, apresentou ganho de peso superior ao dos animais do lote 38, demonstrando que o uso do produto em questão proporcionou ganho superior.

Tabela 5 - Comparação entre os Lotes 34 e 38, Uberlândia, 2008.

\begin{tabular}{llllllllll}
\hline Lote & $\mathbf{D O}$ & $\mathbf{D 3 0}$ & $\mathbf{D 6 0}$ & $\mathbf{G P}$ & $\mathbf{G D}$ & $\mathbf{G L}$ & Custo & $\mathbf{G L D}$ & $\mathbf{G R}$ \\
& $\mathbf{( K g )}$ & $\mathbf{( K g )}$ & $\mathbf{( K g )}$ & $\mathbf{( K g )}$ & $\mathbf{( K g )}$ & $\mathbf{( K g )}$ & $\mathbf{( R \mathbf { } )}$ & $\mathbf{( R \mathbf { } )}$ & $\mathbf{( R \mathbf { ~ } )}$ \\
\hline $\mathbf{3 4}$ & 422,48 & 512,01 & 546,89 & $124,41 \pm 24,17^{a}$ & 7,89 & 4,18 & 30,68 & 25,08 & - \\
& & & & & & & & & 5,44 \\
$\mathbf{3 8}$ & 426,75 & 495,73 & 543,29 & $116,54 \pm 18,83 \mathrm{~b}$ & & 11,04 & & \\
\hline
\end{tabular}

Letras iguais na mesma coluna não diferem entre si pelo teste de Tukey $(p>0,05)$

GP- Ganho de peso; GD- Ganho de peso diário

Custo- Custo dos medicamentos aplicados

GL- Ganho Líquido com 53\% de rendimento de carcaça

GLD- Ganho Líquido em dinheiro (Custo de $\mathrm{R} \$ 90,00$ por arroba)

GR- Ganho Real após a comparação entre os tratamentos (GLD- Diferença entre os custos)

Durante o período experimental os animais que se apresentavam apáticos e com algum problema de saúde foram retirados do experimento e levados para o hospital da propriedade, do lote 38 foram retirados quatro animais, sendo que apenas 1 apresentou quadro pneumonia clínica e foi tratado com Micotil (Elanco), e os demais apresentaram problemas de casco e também foram retirados do experimento. 
BARBOSA, I.L.A. et al. Ganho de peso em bovinos em confinamento utilizando enrofloxacino e polivacinas. PUBVET, Londrina, V. 5, N. 8, Ed. 155, Art. 1049, 2011.

O uso do Catosal nos animais do lote 38 demonstrou que mesmo não havendo diferença estatística entre os ganhos de pesos dos grupos deste lote, ficou observado que o lote que recebeu Catosal no D0 e D30 obteve um ganho $(119,05 \mathrm{Kg})$ contra $(115,41 \mathrm{Kg})$, do lote que recebeu apenas uma aplicação. Para comprovação desta hipótese, outros experimentos serão realizados.

\section{Conclusão}

Ao avaliarmos todos os dados apresentados neste experimento podemos concluir que:

O Enrofloxacino pode ser usado com sucesso como metaprofilático em animais confinados, proporcionando a redução de doenças respiratória e aumentando o ganho de peso dos animais.

O uso de Enrofloxacino no período de adaptação dos animais na entrada do confinamento proporcionou o efeito metaprofilático esperado, uma vez que o número de ocorrências de lesões e sintomas de pneumonia nos animais do lote 34 foram zero, e no lote que não recebeu o produto foi de 3,92\%.

Trabalhos em relação ao custo do produto, principalmente em relação ás embalagens maiores, destinada para o confinamento podem ser realizados no intuito de se reduzir o custo da aplicação, para que a mesma seja viabilizada, já que ficou comprovado que no experimento em questão o produto pode ser usado com sucesso para cumprir o efeito metafilático, e aumentando o ganho de peso final dos animais.

O mesmo sugerido para o produto Enrofloxacino também vale para os demais produtos em questão do pacote Bayer, uma vez que embalagem maior consegue-se uma redução de custo significativa, que poderá viabilizar o custo do pacote sugerido.

Os produtos que mais elevaram no orçamento do pacote Bayer foram o Catosal e o Enrofloxacino.

A avaliação econômica do grupo 1 L-34 em relação ao grupo 3 L-38 reforça e comprova todas as inferências supracitadas, uma vez que o valor financeiro 
BARBOSA, I.L.A. et al. Ganho de peso em bovinos em confinamento utilizando enrofloxacino e polivacinas. PUBVET, Londrina, V. 5, N. 8, Ed. 155, Art. 1049, 2011.

obtido por animal ( $R \$ 13,36)$, este valor foi positivo, além do ganho de peso proporcionado pelos produtos Bayer em avaliação.

\section{Referências}

BEEFPOINT. Pesquisa Top BeefPoint de Confinamentos 2006/2007. Disponível em: www.beefpoint.com.br. Acessado em 28 dez de 2007

BRANDINI, J.C et al. Doença em bovinos confinados. Disponível em: http://www.cnpgc.embrapa.br/publicacoes/doc/doc65. Acesso em 30 out 2008.

CARDOSO, Esther Guimarães. Confinamento de bovinos. Disponível em: http://www.cnpgc.embrapa.br/publicacoes/naoseriadas/cursosuplementacao/confinamento/\#1 . Acesso em 25 out 2008.

CARDOSO, Esther Guimarães. Engorda de bovinos em confinamento. Disponível em: http://www.cnpgc.embrapa.br/publicacoes/doc/doc64/. Acesso em: 25 out 2008.

DUARTE, Marcos. Utilização de subprodutos na alimentação de bovinos em confinamento. Disponível em: http://www.infoescola.com/zootecnia/utilizacao-desubprodutos-na-alimentacao-de-bovinos-em-confinamento. Acesso em 22 out 2008.

EMBRAPA. Engorda de bovinos em confinamento - Aspectos gerais. Disponível em: www.cnpgc.embrapa.br/publicações/doc/doc64/00resumo.html/. Acessado em 04 dez 2008.

FARIA, Thales dos Anjos. Bovino de corte. Disponível em: www.grupocultivar.com.br/artigo.asp?id=794. Acessado em 24 de out 2008.

Kinetomax:

$\underline{w} w$ w.avedomestica.com/index.php?option $=$ com content\&task=view\&id $=2034 \&$ Itemid $=127$

LANGONI, Hélio. Vacinas: Quando e como usá-las. Disponível em: www.spmv.org.br/conpavet2004/palestras $\% 20-\% 20$ resumos/Vacinas $\% 20$ -

\%20quando $\% 20$ e $\% 20$ como $\% 20 \% 20 \% 20 \% 20 \% 20 \% 20$-Helio\%20Langoni.doc. Acessado em 10 nov 2008.

LOBATO, F.C. F; SALVARINI, F.M; ASSIS, K.A. Clostridioses de pequenos ruminantes. Disponível em: www.fmv.utl.pt/spcv/PDF/pdf6_2007/23-34.pdf. Acesso em: 2 Nov 2008.

MARTINI,L.C.T. Confinamento de bovinos de corte. São Paulo; Nobel, 1987.

MELLO, E. Desenvolvimento de bovinos em confinamento com diferentes fontes de alimento. Uberlândia; Faculdade de Medicina Veterinária, 1996. p-41. Trabalho (Graduação em Medicina Veterinária).

MORGADO, José Carlos. Clostridioses: Inimigo "número um" do rebanho. Disponível em: www.revistarural.com.br/Edicoes/2002/Artigos/rev57 clostridioses.htm. Acessado em 1 nov 2008.

RADOSTITS, O. M. et al. Clínica veterinária: Um tratado de doenças dos bovinos, ovino, suínos, caprinos e eqüinos. Rio de Janeiro: Guanabara/Koogan, p.764-767, 200.

SMITH, B. Medicina Interna dos Grandes Animais. 2006. 
BARBOSA, I.L.A. et al. Ganho de peso em bovinos em confinamento utilizando enrofloxacino e polivacinas. PUBVET, Londrina, V. 5, N. 8, Ed. 155, Art. 1049, 2011.

TORRE JR, A. M., NOGUEIRA, M. P. Confinamento - Perspectivas para 1999. Revista Pecuária de Corte. IRAPEC. n.89, p.65-66, 1999. 